\title{
MASS MEASUREMENT OF ACCRETING MAGNETIC WHITE DWARFS WITH HARD X-RAY SPECTROSCOPY
}

\author{
M. ISHIDA AND R. FUJIMOTO \\ Institute of Space and Astronautical Science \\ 3-1-1 Yoshinodai, Sagamihara, Kanagawa 229, JAPAN
}

\section{Introduction}

Accreting magnetic white dwarfs are usually found as component stars in Magnetic Cataclysmic Variables (MCVs), in which a white dwarf with $B=10^{5-8} \mathrm{G}$ accepts mass from a late type (secondary) star via Roche Lobe overflow. Matter from the secondary is funneled by the magnetic field and concentrates on the magnetic pole(s) of the white dwarf. Since the accretion flow becomes highly supersonic, a standing shock wave is formed close to the white dwarf. The temperature of the plasma at the shock front reflects the gravitational potential and can be denoted as a function of the mass $(M)$ and the radius $(R)$ of the white dwarf as:

$$
k T_{\mathrm{S}}=\frac{3}{8} \frac{G M}{R} \mu m_{\mathrm{H}}=22\left(\frac{M}{0.6 M_{\odot}}\right)\left(\frac{R}{8.7 \times 10^{8} \mathrm{~cm}}\right)^{-1} \quad[\mathrm{keV}] .
$$

Note here that the height of the shock is expected to be within $10 \%$ of the white dwarf radius, and hence neglected here.

As the equation of state of the degenerate electron is well studied, $M-R$ relation of the white dwarf is established (Hamada \& Salpeter 1961, Nauenberg 1972). We can thus determine the mass and the radius of the white dwarf if we successfully measure $T_{\mathrm{S}}$ from the $\mathrm{X}$-ray spectroscopy.

However, since the post-shock plasma is cooled by the optically thin thermal plasma emission as it descends the accretion column, the continuum spectrum cannot be represented by a single temperature thermal bremsstrahlung (Aizu 1973). The continuum spectral shape becomes further complicated because of the photoelectric absorption in the pre-shock accretion column. As the photons from the plasma travel along different paths through the accretion column, the absorption cannot be represented by a single column density (Ishida, Fujimoto 1995). In addition, it is also revealed that the reflection from the white dwarf surface makes significant contribution to the observed spectrum. As easily imagined, it is very difficult to evaluate all these effects consistently and derive $T_{\mathrm{S}}$ (although significant progress was made recently; Cropper et al. 1997, Done, Magdziarz 1997). 
In this paper, we present a new method of measuring $T_{\mathrm{S}}$ and hence the mass of the white dwarf by means of the $K \alpha$ emissions lines from abundant heavy elements. The essential ideas presented in $\S 2$ are already summarized in Fujimoto, Ishida (1997) in detail. In $\S 3$, comparison of the masses so far determined with our method to those from optical observations are summarized. Finally, concluding remarks are given in $\$ 4$.

\section{Method and Results}

Since the emission from the post-shock plasma is optically thin, the observed intensity of the $K \alpha$ emission line from the element $Z$ at the ionization state $z$ is expressed as

$$
I_{Z, z}\left(T_{\mathrm{S}}, T_{\mathrm{B}}, A_{Z}, S, D\right)=\frac{S}{4 \pi D^{2}} \int_{T_{\mathrm{B}}}^{T_{\mathrm{S}}} A_{Z} \varepsilon_{Z, z}(T) n(z(T))^{2} \frac{d z}{d T} d T
$$

where $T_{\mathrm{B}}$ is the temperature at the base of the optically thin accretion column, $A_{Z}, S$ and $D$ are the abundance of the element $Z$ in the unit of Solar, cross section of the accretion column, and the distance to the source, respectively. $\varepsilon_{Z, z}(T)$ is the volume emissivity (photons $\mathrm{cm}^{3} \mathrm{~s}^{-1}$ ) of $K \alpha$ line, which is tabulated by Mewe et al. (1985). Density and temperature profiles of the accretion column is calculated by Aizu (1973) by assuming pure thermal bremsstrahlung cooling. Although eq.(2) depends upon several uncertain factors such as $A_{Z}, S$ and $D$, we can eliminate all of them by using the intensity ratio of the hydrogenic to the He-like $K \alpha$ line of the same atom. Since this ratio $R_{Z}=I_{Z, \mathrm{H}} / I_{Z, \mathrm{He}}$ depends only upon $T_{\mathrm{S}}$ and $T_{\mathrm{B}}$, we can constrain them if we can measure $R_{Z}$ for two elements at least.

In Fig. 1, we show the allowed area of $T_{\mathrm{S}}$ and $T_{\mathrm{B}}$ obtained by the $K \alpha$ emission line ratios resolved by the $A S C A$ SIS for the three MCVs - EX Hya, AO Psc and V1223 Sgr. The Masses of the white dwarf determined from eq.(1), referring to the resulting range of $T_{\mathrm{S}}$, are labeled on top of each panel (Fujimoto 1996).

\section{Comparison with Optical Measurements}

We have successfully measured the mass of the white dwarf with the hard X-ray line spectroscopy. Our method just uses the depth of the gravitational potential at the surface of the white dwarf, and hence potentially applicable to all the MCVs. Masses of binary components are usually measured by optical line velocity measurements. This is, however, reliable only for eclipsing systems, because of uncertainty of the inclination angle. Our method is, of course, free from this uncertainty.

To establish reliability of our method, it is necessary to compare the results with those from optical line velocity measurements in eclipsing MCVs. A remarkable agreement is already obtained for EX Hya from which velocity amplitudes of the white dwarf and the secondary are both measured to be $69 \pm 9 \mathrm{~km} \mathrm{~s}^{-1}$ (Hellier et al. 1996) and $356 \pm 4 \mathrm{~km} \mathrm{~s}^{-1}$ (Smith et al. 1993). With these numbers, the mass of the white dwarf in EX Hya is obtained to be $0.49 \pm 0.03 M_{\odot}$, which should be compared with $0.48_{-0.06}^{+0.10} M_{\odot}$ shown in Fig. 1 .

There are two more eclipsing MCVs whose X-ray intensity is strong enough for the line spectroscopy. One of them is XY Ari which is discovered by Ginga 
(a) EX Hya $-M=0.48_{-0.06}^{+0.10} M_{\odot}$

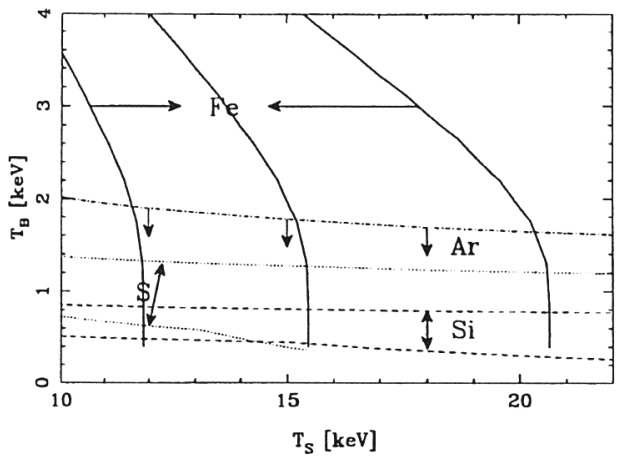

(c) $\mathrm{V} 1223 \mathrm{Sgr}-M>0.82 M_{\odot}$

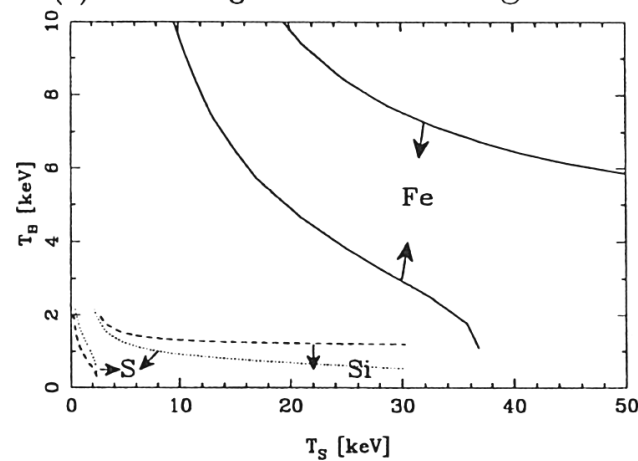

(b) $\mathrm{AO}$ Psc $-M=0.40_{-0.10}^{+0.07} M_{\odot}$

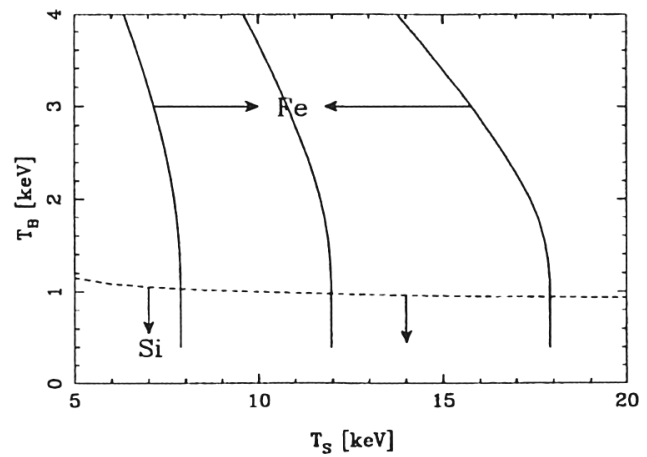

Figure 1. Constraints on $T_{\mathrm{S}}$ and $T_{\mathrm{B}}$ for EX Hya, AO Psc and V1223 Sgr obtained by the line spectroscopy by the $A S C A$ SIS. The allowed area is the overlap of the strips from all the elements. The white dwarf Masses determined from $T_{\mathrm{S}}$, with the aid of the theoretical $M-R$ relation, are labeled at the top of each panel.

(Koyama et al. 1991, Kamata, Koyama 1993). Fig. 2 show (a) the X-ray spectrum obtained by the SIS with roughly $90 \mathrm{ks}$ observation, and (b) the allowed range for $T_{\mathrm{S}}$ and $T_{\mathrm{B}}$. As XY Ari is behind the molecular cloud Lynds 1457 , the low energy $\mathrm{X}$-ray intensity is attenuated. As a result, we can obtain only the line intensity ratio of iron. Assuming that the plasma is cooled in the same manner as in the sources shown in Fig. 1, we have tentatively obtained the mass of the white dwarf in XY Ari to be $1.1_{-0.5}^{+0.3} M_{\odot}$ as shown in Fig. 2(b). Note that the upper boundary is Chandrasekhar limit. On the other hand, there is no optical measurement yet of the mass of the white dwarf in XY Ari. Optical observation is strongly encouraged.

The other candidate MCV is $1 \mathrm{H} 1752-081$ (V2301 Oph), which is strong enough but not observed yet by $A S C A$. The mass of the white dwarf is estimated to be $0.5-0.8 M_{\odot}$ by the optical observation (Silber et al. 1995).

\section{Concluding Remarks}

We have successfully measured the masses of the white dwarf in the three MCVs - EX Hya, AO Psc, V1223 Sgr, and possibly in XY Ari. All these sources belong to so-called intermediate polars, in which the magnetic field of the white dwarf is relatively weak. In polars, which include a stronger field white dwarf, optical cyclotron emission dominates over the thermal bremsstrahlung close to the shock front. In this case, $T_{\mathrm{S}}$ and hence the mass measured from the hard $\mathrm{X}$-ray line 
(a)

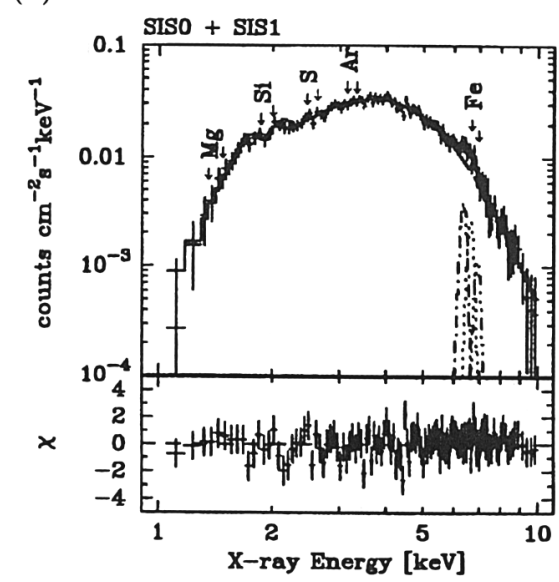

(b)

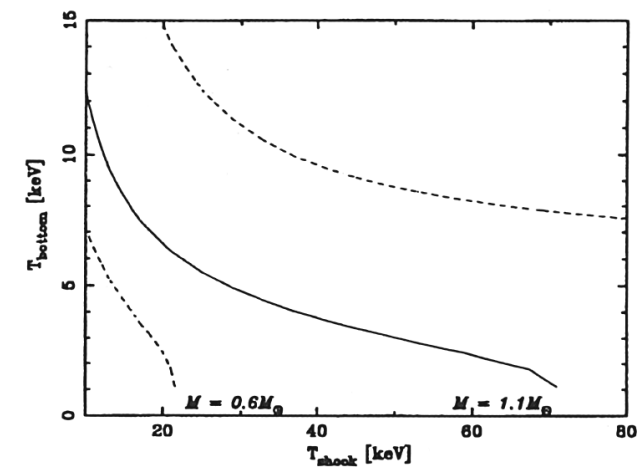

Figure 2. (a) The SIS spectrum of XY Ari, and (b) Allowed range for $T_{\mathrm{S}}$ and $T_{\mathrm{B}}$.

spectroscopy become lower limits to them. To evaluate the contribution of the cyclotron emission relative to the thermal bremsstrahlung, it is necessary to measure the density of the post-shock hot plasma, which will be possible by the ASTRO-E micro-calorimeter.

The other MCVs are generally too faint for $A S C A$ to perform a high quality $\mathrm{X}$-ray spectroscopy. Mass determination of such faint objects with our method will be taken over to the next generation $\mathrm{X}$-ray astronomy satellites - $A X A F, X M M$ and $A S T R O-E$.

\section{References}

Aizu, K. (1973) Prog. Theoret. Phys., 49, 1184

Cropper, M., Ramsay, G. and Wu, K. (1997) MNRAS, in press

Done, C. and Magdziarz, ?? (1997) MNRAS, submitted

Fujimoto, R. and Ishida, M. (1997) $A p J, 474,774$

Fujimoto, R. (1996) Ph.D. Thesis, University of Tokyo

Hamada, T. and Salpeter, E. E. (1961) ApJ, 134, 683

Hellier, C. (1996) in IAU Colloq. 158, Cataclysmic Variables and Related Objects, eds. A. Evans and J. H. Wood, Kluwer, Dordrecht, p. 143

Ishida, M. and Fujimoto, R. (1995) in Cataclysmic Variables, eds. A. Bianchini, M.DellaValle, M. Orio, Kluwer, Dordrecht, p. 93

Kamata, Y. and Koyama, K. (1993), ApJ, 405, 307

Koyama, K., Takano, S., Tawara, Y., Matsumoto, K., Noguchi, K., Fukui, Y., Iwata, T., Ohashi, N., Tatematsu, K., Takahashi, N., Umemoto, T., Hodapp, K. W., Rayner, J. and Makishima, K. (1991) ApJ, 377, 240

Mewe, R., Gronenschild, E. H. B. M. and van den Oord, G. H. J. (1985) A\&A Suppl., 62, 197

Nauenberg, M. (1972) $A p J, 175,417$

Silber, A. D., Remillard, R. A., Horne, K. and Bradt, H. V., (1994) ApJ, 424, 955

Smith, R. C., Collier-Cameron, A. and Tucknott, D. S. (1993) in Cataclysmic Variables and Related Objects, eds. O. Regev and G. Shaviv (Ann. Israel Phys. Soc., Vol.10). p. 70 